\title{
Effect of Longitudinal Temperature Gradient on the Intensity of Two-Way Evaporation in Axisymmetric Capillaries
}

\author{
V.L. Malyshev ${ }^{1, *}$ \\ ${ }^{1}$ Mogilev state University of Foodstaffs, BY-212027, Mogilev, Belarus
}

\begin{abstract}
A two-way evaporation of liquids from conical channels has been studied . The influence of the temperature gradient on the mass transfer process has been considered. An approximation expression for the temperature coefficient of evaporation has been derived and algorithm for calculating the system drainage time is developed. The double-sided vaporization from conical channels at an axial temperature gradient has been investigated in detail. Expressions for temperature coefficient of evaporation has been obtained and a computational algorithm for vaporization velocity has been developed. The obtained results allow estimating the duration of drying for materials with through pores of any type under conditions of uneven heating.
\end{abstract}

\section{Introduction}

In real mass transfer processes due to uneven heating of the drained materials, as a rule, there are temperature gradients. In this regard, accounting for non-isothermal effects in mass transfer processes is an urgent task.

Thus, the previously solved problem [1], in which evaporation from capillaries occurs simultaneously from different sides, is complicated by the presence of a temperature gradient along the channel axis.

The solution of the problem of liquid evaporation from the diffuser channel corresponds to mass transfer through the wide end of axisymmetric channels with radius $r_{01}$, and evaporation from the confusor channels corresponds to mass transfer through the narrow end of the channel with radius $r_{02}$.

Axisymmetric channels whose radii along the center line can be given by formulae (1) - (3) are considered as working models:

$$
\begin{array}{r}
r(z)=r_{0}[1+(a-1) z], \quad \text { (model I) } \\
r(z)=r_{0} a^{z}, \quad \text { (model II) } \\
r(z)=r_{0} \sqrt{1+\left(a^{2}-1\right) z} \text {. (model III) }
\end{array}
$$

To characterize them, a dimensionless parameter a is introduced, which determines the ratio of limit radii $\mathrm{R}_{0} / \mathrm{r}_{0}$, where $\mathrm{r}_{0}$ is the initial radius $(x=0), R_{0}$ the final radius $(x=L)$. It is obvious that for cylindrical channels it is equal to one. Porous materials that have capillaries that have holes on the surface of a larger radius than in the depth of the body are called diffusers $(a<1)$ structures with holes on the surface of a smaller transverse size than in the volume $(a>1)$

Changes in the radius of the capillary are determined by a dimensionless parameter $b=\frac{d r}{d x}$, called taper [2]. The differences between the varieties of capillaries associated with unequal axis $\mathrm{x}$ curvature of the walls $k=\frac{d b}{d x}$

The change in the cross-sectional area along the axis x can occur both uniformly $(k=0)$ and unevenly-first slowly, then faster $(k>0)$, or vice versa $(k<0)$. 


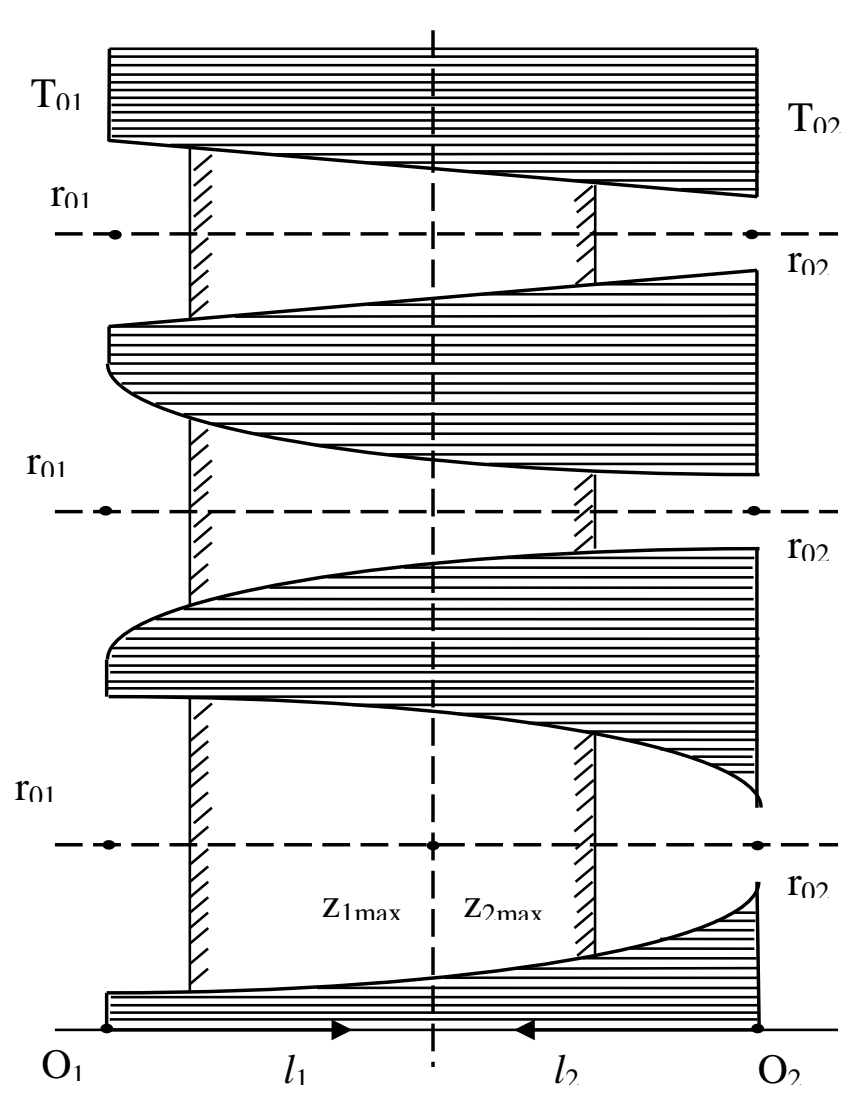

Fig.1. Scheme of evaporation from a porous film at uneven heating $(\mathrm{T} 01 \neq \mathrm{T} 02)$. Models of through pores are numbered from top to bottom (I, II, III). Indices of wide mouths - 1, 2, 3, narrow, respectively, $-4,5,6$.

\section{General solution of the problem of oncoming movement of meniscus in confusor and diffusor parts of conical capillaries}

The evaporation time of the liquid through the mouths in model I $\left(a_{1}<1, k_{1}=0\right)$ and $\left(a_{2}>1, k_{2}=0\right)$ is determined by the integration of the equations [3]

$$
\begin{array}{r}
\frac{z_{1} d z_{1}}{\left[1+\left(a_{1}-1\right) z_{1}\right]^{2}}=\gamma_{01}^{2} B_{1}(T) F d t, \\
{\left[1+\left(a_{2}-1\right) z_{2}\right]^{2} z_{2} d z_{2}=\gamma_{02}^{2} B_{2}(T) F d t .}
\end{array}
$$

For model II, the time of mass transfer through opposite mouths $\left(a_{1}<1, k_{1}<0\right.$ и $\left.a_{2}>1, k>0\right)$ is determined by the equations [3]

$$
\begin{aligned}
& \frac{z_{1} d z_{1}}{a_{1}^{2 z_{1}}}=\gamma_{01}^{2} B_{1}(T) F d t, \\
& a_{2}^{2 z_{2}} z_{2} d z_{2}=\gamma_{02}^{2} B_{2}(T) F d t
\end{aligned}
$$

The evaporation time of the liquid through the mouths of the model III $\left(a_{1}<1, k_{1}>0\right.$ и $\left.a_{2}>1, k_{2}<0\right)$ is determined by the integration of the equations [3]

$$
\begin{aligned}
& \frac{z_{1} d z_{1}}{\left[1+\left(a_{1}^{2}-1\right) z_{1}\right]}=\gamma_{01}^{2} B_{1}(T) F d t, \\
& z_{2}\left[1+\left(a_{2}^{2}-1\right) z_{2}\right] d z_{2}=\gamma_{02}^{2} B_{2}(T) F d t
\end{aligned}
$$

Two independent processes can be combined by synchronizing them. For each model, equating the evaporation time on each side in pairs, it is possible equations as an upper limit. In the system with them, one should consider a condition $Z_{1 \max }+Z_{2 \max }=1$, that allows one of the unknowns to be excluded from consideration $-Z_{1 \max }$ or $Z_{2 \max }$. A necessary condition for the integration of the obtained equations is the representation of the function $B(T)$ in an analytical form, for which the corresponding approximation expressions should be obtained on the basis of reference data $[4,5]$.

The following designations are used: $\gamma_{0 i}=r_{0 i} / L$. relative radius of the $\mathrm{i}$-th mouth $(\mathrm{i}=1$ - diffuser, $\mathrm{i}=2$ confuser); $z_{i}=l_{i} / L$ - relative coordinate of the $\mathrm{i}$-th meniscus; $l_{i}$ - position of the i-th meniscus in the channel length $\mathrm{L} ; \quad B_{1}=\frac{\left(c_{S}^{2}-1\right)}{\eta T}-$ temperature coefficient of evaporation, $c_{S}=\frac{P_{S}}{P_{0}}$ - relative concentration of saturated steam, $F=\frac{P_{0}^{2} \mu}{16 R \rho}$ - molecular coefficient of evaporation; $P_{S_{-}}$pressure of saturated steam; $\mathrm{T}-$ absolute temperature; t-time; $P_{0}$ - external pressure; $\mu$ - molar mass of the evaporated liquid; $\rho_{\text {- density of }}$ the liquid; R- universal gas constant; $\eta_{-}$coefficient of dynamic viscosity of the vapor-gas mixture, which was calculated as a value averaged by partial pressures [6]

$$
\eta=\frac{\eta_{1} P_{S}+\eta_{2} P_{0}}{P_{S}+P_{0}}=\frac{\eta_{1} c_{S}+\eta_{2}}{c_{S}+1} .
$$

\section{Determining the coordinates of the meeting of the menisci}

In solving the problem, the thermodiffusion contribution to mass transfer due to its smallness in relation to the viscous flow [7] was not taken into account.

Let us turn to the case of evaporation of a liquid heated above the boiling point. As the experimental data show, with increasing temperature, the forces of interaction between the liquid molecules and the capillary walls rapidly decrease and become negligible at 
$T>340 K$ (for water and quartz surface) [8]. This allows us to exclude capillary forces from consideration, to consider evaporation in opposite directions as independent, and to consider the liquid - vapor interface as flat.

Assume, that the temperature change along the channel axis runs in a straight line. For certainty, assume, that the temperature $T_{1}$ at the wide mouth exceeds the temperature $T_{2}$ at the narrow. We believe that the considered temperature range corresponds to the condition $\mathrm{T}>\mathrm{Tboil}$. Therefore, in any part of the capillary evaporation from the surface occurs in a viscous mode. However, in the non-isothermal case, any cross-section of the channel will correspond to its own temperature, which means different numerical values of the temperature coefficient of evaporation $B(T)$. Since the temperature changes along the axis of the channel, the coefficient $\mathrm{B}$ is dependent on the coordinate of the meniscus.

To integrate the obtained expressions, one should , based on the approximation formula for $B(T)$, find an explicit form of the function $T(z)$.

As a working variant, we take the dependence $B(T)$ in the form applicable in the temperature range $377 \div 413 K$ :

$$
B(T)=\alpha T^{2}-\beta T+\varepsilon,
$$

where

$$
\begin{gathered}
\alpha=1,24624\left[\kappa 2 /\left(\mu c^{2} K\right)\right] ; \\
\beta=933,393\left[\kappa 2 /\left(\mu c^{2}\right)\right] ; \\
\varepsilon=175033,27896\left[\kappa 2 K /\left(\mu c^{2}\right)\right] .
\end{gathered}
$$

In the case of the assumed linear decrease in temperature from $T_{01}$ to $T_{02}$ for the mouths of the diffuser type we obtain

$$
T_{1}=T_{01}-\left(T_{01}-T_{02}\right) z_{1} .
$$

Then the dependence $B_{1}\left(z_{1}\right)$ takes the form of

$$
B_{1}\left(z_{1}\right)=A z_{1}^{2}+D_{1} z_{1}+C_{1},
$$

where

$$
\begin{gathered}
A=\alpha\left(T_{01}-T_{02}\right)^{2}\left[\kappa 2 /\left(\mu c^{2} K\right)\right] \\
D_{1}=-\left(2 \alpha T_{01}+\beta\right)\left(T_{01}-T_{02}\right)\left[\kappa 2 /\left(\mu c^{2}\right)\right] ; \\
C_{1}=\left(\alpha T_{01}^{2}+\beta T_{01}+\varepsilon\left[\kappa 2 K /\left(\mu c^{2}\right)\right]\right) .
\end{gathered}
$$

For the mouths of the confusor type we have

$$
\begin{gathered}
T_{2}=T_{02}+\left(T_{01}-T_{02}\right) z_{2} \\
B_{2}\left(z_{2}^{2}\right)=A z_{2}^{2}+D_{2} z_{2}+C_{2} ; \\
A=\alpha\left(T_{01}-T_{02}\right)^{2} ; \\
D_{2}=\left(2 \alpha T_{02}+\beta\right)\left(T_{01}-T_{02}\right) ; \\
C_{2}=\left(\alpha T_{02}^{2}+\beta T_{02}+\varepsilon\right) .
\end{gathered}
$$

The laws of motion of interfacial surfaces for diffuser channels have the form (4),(6),(8) $\left(a_{1}<1\right)$. For capillaries with confusor-type mouths $\left(a_{2}>1\right)$, the equations of motion of the meniscus are written as (5),(7),(9).

It should be remembered that $a_{2}=\frac{1}{a_{1}}$, since

$$
7_{1} a_{1}=\frac{r_{02}}{r_{01}} \text { and } a_{2}=\frac{r_{01}}{r_{02}} \text {. }
$$

We will synchronize the evaporation processes on both sides of the channels of the same type:

$\mathrm{j}=1$ :

$$
\int \frac{z_{1} d z_{1}}{a_{1}^{2 z_{1}}\left(A_{1} z_{1}^{2}+D_{1} z_{1}+C_{1}\right)}=F \gamma_{01}^{2} \int d t
$$

(model I)

$j=4:$

$$
\int \frac{a_{2}^{2 z_{2}} \cdot z_{2} d z_{2}}{\left(A z_{2}^{2}+D_{2} z_{2}+C_{2}\right)}=F \gamma_{02}^{2} \int d t
$$

(model I)

$j=2$ :

$$
\int \frac{z_{1} d z_{1}}{\left(1+W_{1} z_{1}\right)^{2}\left(A z_{1}^{2}+D_{1} z_{1}+C_{1}\right)}=F \gamma_{01}^{2} \int d t
$$

(model II)

$j=5$ :

$$
\int \frac{\left(1+W_{2} z_{2}\right)^{2} z_{2} d z_{2}}{\left(A z_{2}^{2}+D_{2} z_{2}+C_{2}\right)}=F \gamma_{02}^{2} \int d t \text {; }
$$

(model II)

$j=3$ :

$\int \frac{z_{1} d z_{1}}{\left(1+W_{3} z_{1}\right)^{2}\left(A z_{1}^{2}+D_{1} z_{1}+C_{1}\right)}=F \gamma_{01}^{2} \int d t ;$ 


$$
\text { (model III) }
$$

$\mathrm{j}=6$ :

$$
\begin{gathered}
\int \frac{\left(1+W_{4} z_{2}\right)^{2} z_{2} d z_{2}}{\left(A z_{2}^{2}+D_{2} z_{2}+C_{2}\right)}=F \gamma_{02}^{2} \int d t \\
\text { (model III) } .
\end{gathered}
$$

The result of integration of the left parts of the equations in the range from 0 to $Z_{1 \max }$ or $Z_{2 \max }$ is denoted by $Y_{j}$, where $j-1 \div 6$, is the model number of one of the mouths of the studied channels.

The coordinate of the meeting of the meniscus in two-way evaporation from the channels of three types is determined from the solution of algebraic equations

$$
\begin{aligned}
& \left.\gamma_{01}^{-2} Y_{1}\right|_{0} ^{z_{\max }}=\left.\gamma_{02}^{-2} Y_{4}\right|_{0} ^{z_{2 \max }}, \text { (model I) } \\
& \left.\gamma_{01}^{-2} Y_{2}\right|_{0} ^{z_{1 \max }}=\left.\gamma_{02}^{-2} Y_{5}\right|_{0} ^{z_{2 \max }}, \text { (model II) } \\
& \left.\gamma_{01}^{-2} Y_{3}\right|_{0} ^{z_{1 \max }}=\left.\gamma_{02}^{-2} Y_{6}\right|_{0} ^{z_{2 \max }}, \text { (model III) }
\end{aligned}
$$

obtained by equating the evaporation time through both mouths of one of the models. Given that the relative length of the channel $Z=1$ and $Z_{2 \max }=1-z_{1 \max }$, exclude from consideration one of the unknowns $\left(z_{2 \max }\right)$. Then the solution of the problem of determining the coordinate of the meeting of the meniscus is to calculate the desired value $Z_{1 \max }$ from the equations

$$
\begin{aligned}
& \left.\gamma_{01}^{-2} Y_{1}\right|_{0} ^{z_{\max }}=\left.\gamma_{02}^{-2} Y_{4}\right|_{0} ^{1-z_{1 \max }},(\text { model I) } \\
& \left.\gamma_{01}^{-2} Y_{2}\right|_{0} ^{z_{1 \max }}=\left.\gamma_{02}^{-2} Y_{5}\right|_{0} ^{1-z_{1 \max }}, \text { (model II) } \\
& \left.\gamma_{01}^{-2} Y_{3}\right|_{0} ^{z_{1 \max }}=\left.\gamma_{02}^{-2} Y_{6}\right|_{0} ^{1-z_{1 \max }} . \text { (model III) }
\end{aligned}
$$

\section{Method of calculation of drying time}

The found limits of integration $Z_{1 \max }$ and $Z_{2 \max }$ allow to calculate the total time of channel drainage ( $t_{01}$ - model I, $t_{02}$ - model II, $t_{03}$ - model III) for the accepted approximation from the equations

(15) or (16) - model I,

(17) or (18) - model II,

(19) or (20) - model III, namely:

$$
\begin{gathered}
t_{01}=\left.F^{-1} \cdot \gamma_{01}^{-2} \cdot Y_{1}\right|_{0} ^{z_{\max }} \\
t_{01}=\left.F^{-1} \cdot \gamma_{02}^{-2} \cdot Y_{4}\right|_{0} ^{z_{2 \max }}-\text { for model I, } \\
t_{02}=\left.F^{-1} \cdot \gamma_{01}^{-2} \cdot Y_{2}\right|_{0} ^{z_{1 \max }} \\
t_{02}=\left.F^{-1} \cdot \gamma_{02}^{-2} \cdot Y_{5}\right|_{0} ^{z_{2 \max }}-\text { for model II, } \\
t_{03}=\left.F^{-1} \cdot \gamma_{01}^{-2} \cdot Y_{3}\right|_{0} ^{z_{1 \max }} \\
t_{03}=\left.F^{-1} \cdot \gamma_{02}^{-2} \cdot Y_{6}\right|_{0} ^{z_{2 \max }}-\text { for model III. }
\end{gathered}
$$

\section{Conclusion}

The results obtained here allow us to estimate the duration of drying of materials with through pores of any fundamentally possible type, under conditions of uneven heating.

\section{References}

1. V.L. Malyshev, Fundamental phys.-math. problems and tech.-technol. systems simulation, 18, 75-84 (2017)

2. N.I. Gamayunov, V.L. Malyshev, L.A. Uvarova, A.S. Feldblyum, J. of Eng. Phys., 47(4), 647-651 (1984)

3. V.L. Malyshev, Proceedings of Belarus NAS, ser.phys.-tech. sciences, 1, 71-76 (2010)

4. M.P. Vukalovich, S.L. Rivkin, A.A. Alexandrov, Tables of water and watering vapoure thermophysical characteristics (M.: Standarts Publ., 1969)

5. N.B. Vargaftic, Directory at thermophysical properties of gases and fluids (M.: Sciences Publ., 1972)

6. M.N. Gaydukov, N.V. Churaev, Yu.I. Yalamov, J. Tech. Phys., 46(10), 2142-2147 (1976)

7. N.I. Gamayunov, A.A. Lankov, High Temperature, 23(4), 781-786 (1985)

8. G.F. Yershova, Z.M. Zorin, N.V. Churaev, Colloid journal, 37(1), 208-210 (1975) 